\title{
Entropic force between biomembranes
}

\author{
Long $\mathrm{Li}^{1,2}$ - Fan Song ${ }^{1,2}$
}

Received: 11 March 2016 / Revised: 5 May 2016 / Accepted: 12 May 2016 / Published online: 11 August 2016

(C) The Chinese Society of Theoretical and Applied Mechanics; Institute of Mechanics, Chinese Academy of Sciences and Springer-Verlag Berlin Heidelberg 2016

\begin{abstract}
Undulation force, an entropic force, stems from thermally excited fluctuations, and plays a key role in the essential interactions between neighboring surfaces of objects. Although the characteristics of the undulation force have been widely studied theoretically and experimentally, the distance dependence of the force, which constitutes its most fundamental characteristic, remains poorly understood. In this paper, first, we obtain a novel expression for the undulation force by employing elasticity and statistical mechanics and prove it to be in good agreement with existing experimental results. Second, we clearly demonstrate that the two representative forms of the undulation force proposed by Helfrich and Freund were respectively the upper and lower bounds of the present expression when the separation between membranes is sufficiently small, which was intrinsically different from the existing results where Helfrich's and Freund's forms of the undulation force were only suitable for the intermediate and small separations. The investigations show that only in a sufficiently small separation does Helfrich's result stand for the undulation force with a large wave number and Freund's result express the force with a small wave number. Finally, a critical acting distance of the undulation force, beyond which the entropic force will rapidly decay approaching zero, is presented.
\end{abstract}

Fan Song

songf@lnm.imech.ac.cn

1 State Key Laboratory of Nonlinear Mechanics (LNM) and Beijing Key Laboratory of Engineered Construction and Mechanobiology, Institute of Mechanics, Chinese Academy of Sciences, Beijing 100190, China

2 School of Engineering Science, University of Chinese Academy of Sciences, Beijing 100049, China
Keywords Undulation force - Flexible membrane - Entropic force $\cdot$ Confined fluctuations

\section{Introduction}

There always exist various attractive and repulsive forces that mediate between neighboring surfaces of objects, in which the van der Waals and undulation force are mainly responsible for the attractive and repulsive forces, respectively $[1,2]$. Because the value of the undulation force is usually very small [2], its effect will efficiently emerge when the scales of objects are smaller than a micron. However, the undulation force usually plays a key role in the interactions between neighboring surfaces of microscale objects. Earlier studies showed that the undulation force governs the various cellular processes, for example, sperm-egg fusion and cell adhesion, binding-unbinding transition, and self-assembly [1-8]; affects the structural stability of liquid crystals $[9,10]$, surfactants [11], and DNA [12]; regulates the mechanical behaviors of polymers [13], proteins [14], gels [15], and graphemes $[16,17]$. In addition, the undulation force can affect the binding affinity of membrane-anchored proteins [18] and the self-assembly of polymers [19].

The concept and quantitative nature of the undulation force were proposed by Helfrich [20] in his original and pioneering work nearly four decades ago. Helfrich approximately treated a biomembrane as lots of very small elements that fluctuated independently, like the molecules of an ideal gas. He asserted that the undulation force essentially varies with $c^{-3}$, where $c$ is the separation between neighboring membranes [20]. Although some assumptions and simplifications were introduced in Helfrich's investigations [20,21], his results were still supported by succeeding studies, including theoretical $[1,22-25]$ and experimental work $[11,26]$, as 
well as computational simulations [27,28]. Recently, Freund [29] reexamined the entropic force under conditions in which some of Helfrich's assumptions [20] were abandoned. Based on the theories of elasticity and statistical mechanics, Freund obtained a set of equations where the undulation force was satisfied. It was regretful that he gave no integral expression of the undulation force because he did not determine the bounds of the transverse deflection of biomembranes. However, employing the asymptotic properties of the equations, Freund [29] found that the undulation force varied with $c^{-1}$ over a large range of separations. This result was in stark contrast to Helfrich's well-accepted result, immediately triggered a series of disputes [2,29-35]. More recently, many studies have presented deep discussions on the difference between the two results and examined the distance dependence of the undulation force. Auth and Gompper [32] and Hanlumyuang et al. [33] divided the acting distance of the undulation force into three regions. Based on Monte Carlo simulations, they proposed that Helfrich's result remained valid for intermediate separations, while the undulation force $f$ roughly satisfied $f \sim 1 /\left(c a^{2}\right)$ for small separations. Therefore, the undulation force was deemed to have a $c^{-1}$ dependence in small separations. Clearly, the role of the lattice constant, $a$, which discretized a membrane in the Monte Carlo simulations, was neglected in the distance dependence of the force. The transition length between the two regions was given as $d_{\text {tran }} \approx a\left(k_{\mathrm{B}} T / \kappa\right)^{1 / 2}$, which was also associated with the discretized length; here, $\kappa$ is the bending rigidity of the membrane. This implied that determining the value of the undulation force heavily relies on the preset parameters in the simulations. For larger separations, Auth and Gompper [32] treated the entire membrane as a soft particle and obtained a $c^{-1}$ force law for separations larger than the crossover length, $0.4 L\left(k_{\mathrm{B}} T / \kappa\right)^{1 / 2}$, while Hanlumyuang et al. [33] showed a $c^{-\eta}$ dependence for the force, in which both the power $\eta$ and the transition length to $c^{-\eta}$ dependence were not integrally given. This is an indication that the distance dependence of the undulation force remains poorly understood.

In the present study, based on the theories of elasticity and statistical mechanics, we obtain an exact integral expression of the undulation force with respect to the separation between membranes and show it to be in good agreement with existing experimental data. Analyzing the expression of the undulation force, we demonstrate that the two representative forms of the undulation force proposed by Helfrich and Freund are not in contradiction to each other. When the separation between membranes is sufficiently small, Helfrich's result shows the undulation force on the membrane with a large wave number, while Freund's result gives the undulation force on the membrane with a small wave number. The two forms of undulation force correspond respectively to the upper and lower bounds of the present result when the separation is sufficiently small. Finally, a critical acting distance of the undulation force, beyond which the entropic force will rapidly decay as it approaches zero, is presented.

\section{Model of undulation force}

To investigate the undulation force and its properties, we focus on a single flexible membrane confined between two parallel rigid planes of spacing $2 c$, as done by Helfrich and Servuss [20,21] and Freund [29]. This is an appropriate model that can be used to study the interactions between membranes in a stack; in terms of a stack with a large number of membranes, the behavior of any single membrane far from either end of the stack exhibits reflective symmetry with respect to its reference plane, and the behavior of neighboring membranes also exhibits reflective symmetry with respect to a plane midway between the reference planes of the two membranes $[20,21,29]$. We assume that the membrane satisfies the periodic boundary conditions on the edges and its reference plane occupies the portion $0 \leq x \leq L, 0 \leq y \leq L$ of the $x y$-plane (Fig. 1).

The transverse deflection of the membrane related to the reference plane is assumed to be $h(x, y)$, which obviously satisfies the confined condition:

$-c \leq h(x, y) \leq c$.

We write the transverse deflection as a complex wave [20, 36]:

$h(\boldsymbol{r})=\sum_{\boldsymbol{q}} h_{\boldsymbol{q}} \exp (\mathrm{i} \boldsymbol{q} \boldsymbol{r})$

where $\mathrm{i}$ is the imaginary unit, $\boldsymbol{q}=(\pi / L)(m, n)$ and $\boldsymbol{r}=$ $(x, y)$ are the wave and coordinate vectors of the membrane, respectively, and the amplitude of the wave satisfies $h_{-q}=$ $h_{q}^{*}$, in which the star denotes complex conjugation. Here $m$ and $n$ are integral numbers, and physically, $|m|=L / \lambda_{x}$ and $|n|=L / \lambda_{y}$ respectively correspond to the wave numbers of the thermal fluctuation modes of the membrane in the $x$ - and $y$-directions, and $\lambda_{x}$ and $\lambda_{y}$ are the wavelengths in the $x$ and $y$-directions, respectively. Further, we extend $h(r)$ to the domain of $-L \leq x, y \leq L$ such that $h(\boldsymbol{r})$ satisfies Eq. (2) in

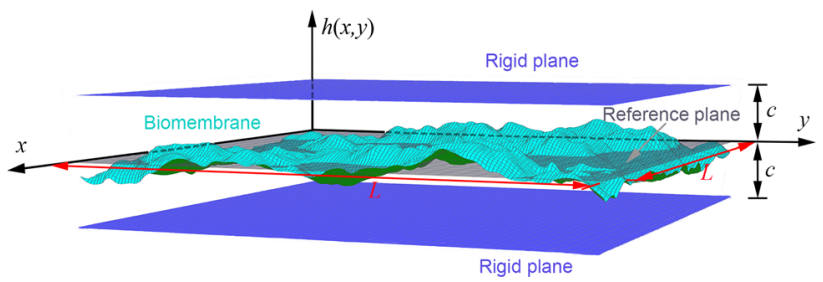

Fig. 1 Schematic diagram showing a thermally fluctuating square biomembrane with dimension of $L \times L$ confined between two rigid planes of separation $2 c . h(x, y)$ is the transverse deflection of the membrane related to the reference plane positioned in $x y$-plane of coordinates 
$0 \leq x \leq L, 0 \leq y \leq L$, and $h(\boldsymbol{r})=0$ otherwise. Thus, the amplitude of the wave is given by the Fourier integral

$h_{\boldsymbol{q}}=\frac{1}{4 L^{2}} \int_{-L}^{L} \int_{-L}^{L} h(\boldsymbol{r}) \exp (-\mathrm{i} \boldsymbol{q} \boldsymbol{r}) \mathrm{d} x \mathrm{~d} y$.

Noting $\left|h_{\boldsymbol{q}}\right|^{2}=h_{\boldsymbol{q}} h_{\boldsymbol{q}}^{*}=h_{\boldsymbol{q}} h_{-\boldsymbol{q}}$ and substituting Eq. (1) into Eq. (3) yields the bounds of the amplitudes

$\left|h_{\boldsymbol{q}}\right|^{2} \leq \frac{c^{2}}{\pi^{4} m^{2} n^{2}}, \quad m, n= \pm 1, \pm 3, \pm 5, \ldots$

The total elastic bending energy of the membrane is written

$$
\begin{aligned}
U & =\int_{0}^{L} \int_{0}^{L} \varepsilon \mathrm{d} \boldsymbol{r}=\sum_{\boldsymbol{q}} \frac{1}{2} \kappa L^{2} q^{4}\left|h_{\boldsymbol{q}}\right|^{2}, m, n= \pm 1, \pm 3, \pm 5, \ldots \\
& =\sum_{\boldsymbol{q}} 2 \kappa L^{2} q^{4}\left|h_{\boldsymbol{q}}\right|^{2}, \quad m, n=1,3,5, \ldots
\end{aligned}
$$

where $\varepsilon=(\kappa / 2)\left(\partial^{2} h / \partial x^{2}+\partial^{2} h / \partial y^{2}\right)^{2}$ denotes the elastic bending energy per unit area of the membrane [20].

According to the theory of statistical mechanics, the thermodynamic partition function of a membrane is

$$
\begin{aligned}
Z & =\int_{0}^{\frac{c}{\pi^{2} L}} \cdots \int_{0}^{\frac{c}{\pi^{2} m n L}} \exp ^{-\beta U} \mathrm{~d}\left(\frac{\left|h_{(1,1)}\right|}{L}\right) \cdots \mathrm{d}\left(\frac{\left|h_{(m, n)}\right|}{L}\right) \\
& =\prod_{m, n} \frac{c}{2 \sqrt{\pi^{3}} m n L \omega_{m n}} \operatorname{erf}\left(\omega_{m n}\right), \quad m, n=1,3,5, \ldots,
\end{aligned}
$$

where $\beta=1 /\left(k_{\mathrm{B}} T\right), k_{\mathrm{B}}$ is the Boltzmann constant, $T$ is the absolute temperature, $\operatorname{erf}(\xi)=(2 / \sqrt{\pi}) \int_{0}^{\xi} \exp ^{-\vartheta^{2}} \mathrm{~d} \vartheta$ is the error function, and $\omega_{m n}$ is a dimensionless factor

$\omega_{m n}=\frac{m^{2}+n^{2}}{m n} \frac{\sqrt{2 \kappa \beta} c}{L}$.

Based on Eq. (6), the free energy of the membrane is expressed as

$$
\begin{aligned}
F= & -\frac{1}{\beta} \ln (Z) \\
= & -\sum_{m, n} \frac{1}{\beta} \ln \left(\frac{c}{2 \sqrt{\pi^{3}} m n L \omega_{m n}} \operatorname{erf}\left(\omega_{m n}\right)\right), \\
& m, n=1,3,5, \ldots
\end{aligned}
$$

Thus, the entropic force acting on the membrane is obtained as

$$
f=-\frac{\partial F}{\partial c}=\frac{2}{\sqrt{\pi} \beta c} \sum_{m, n} \Phi\left(\omega_{m n}\right), \quad m, n=1,3,5, \ldots,
$$

where

$\Phi\left(\omega_{m n}\right)=\frac{\omega_{m n} \exp \left(-\omega_{m n}^{2}\right)}{\operatorname{erf}\left(\omega_{m n}\right)}$

Obviously, Eq. (9) gives an integral expression of the undulation force exerted on a membrane and shows that the distance dependence of the force is strongly nonlinear and very complex, which is intrinsically different from existing theoretical and computational results. This is because $\omega_{m n}$ in Eq. (9) explicitly includes the separation between membranes, $c$, as indicated in Eq. (7). Simultaneously, Eq. (9) is also dependent strongly on the wave numbers of the membrane, $m$ and $n$.

In fact, the thermally excited membrane fluctuation stems intrinsically from the stimulation of Brownian motion of solvent molecules, which results in there being no preferential orientation of the undulation force acting in the plane of the membrane with the thermally excited fluctuation. Therefore, the wavelength of the waves generated by thermal excited fluctuation in the $x$ - and $y$-directions in the plane of the membrane itself should be equal statistically to one another. In terms of the square membrane studied here, the wave numbers of the membrane in the $x$ - and $y$-directions, $m$ and $n$, should be equal statistically to one another.

According to Eq. (7), when $m=n, \omega_{m n}$ is written as

$\omega=\frac{2 \sqrt{2 \kappa \beta} c}{L}$.

Substituting Eq. (11) into Eq. (9), the undulation force is written as

$f=\frac{2(2 k-1)^{2}}{\sqrt{\pi} \beta c} \frac{\omega \exp \left(-\omega^{2}\right)}{\operatorname{erf}(\omega)}, \quad k=1,2,3, \ldots$,

where $k$ is associated with the wave number of the membrane and $m=n=2 k-1$. With an increase in $k$, the undulation force in Eq. (12) also increases monotonically. This is an indication that the wave number yielded in a membrane plays a key role in the undulation force generated on the membrane. In addition, because $\Phi\left(\omega_{m n}\right)$ is a monotonically decreasing function with respect to $\omega_{m n}$ from Eq. (10), Eq. (12) in fact stands for the minimum curve of Eq. (9) and appropriately expresses the undulation force exerted on a square membrane.

Comparing the results of Eq. (12) with the existing experimental data [26], we find that the present results are in good agreement with the experimental results (Fig. 2). 


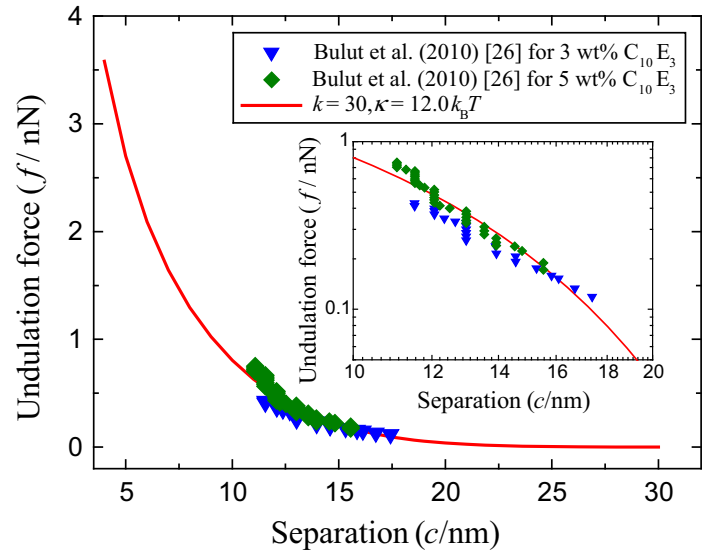

Fig. 2 Computational plots showing distance dependence of undulation force; solid lines: results of Eq. (12) for $T=300 \mathrm{~K}$ and $L=1.0 \mu \mathrm{m}$; points are derived from the experimental data from Ref. [24]; inset displays detail of comparison between present and experimental results

\section{Properties of undulation force}

We now investigate the two extreme cases of the undulation force in Eq. (12). First, when a membrane only generates a single wave under the undulation force, the wavelength of the wave will be at the maximum value, $\lambda_{x}=\lambda_{y}=\lambda_{\max }=L$. Thus, the wave number of the membrane is at its minimum value, $m_{\min }=n_{\min }=k_{\min }=L / \lambda_{\max }=1$. At this time, the undulation force stands for the minimum value of Eq. (12), which is expressed as

$f_{\min }=\frac{2}{\sqrt{\pi} \beta c} \frac{\omega \exp \left(-\omega^{2}\right)}{\operatorname{erf}(\omega)}$.

In particular, if the variation $\omega$ is sufficiently small, $\omega \ll 1$, i.e., the separation between the membranes is very small, $c \ll L$, then Eq. (13) is reduced to

$f_{\min }=\frac{1}{\beta c}$.

Equation (14) shows that the undulation force varies with $c^{-1}$ when $c \ll L$, which is identical to the result given by Freund [29] and is in agreement with the results of Auth and Gompper [32] and Hanlumyuang et al. [33] for small separations. Obviously, Eq. (14) forms the lower bound of Eq. (12) when the separation is sufficiently small (Fig. 3).

Note that if the membrane is perfectly rigid, its wave number is equal to one. Equation (13) obviously includes the undulation force exerted on the rigid membrane.

Second, we consider that the dimensions of the membrane approach a single molecular scale. In this case, the membrane is similar to a single molecule that keeps random thermal vibrations in the direction normal to the reference plane of the membrane. At this time, both the wavelength of the membrane in the $x$ - and $y$-directions are at their minimum values,

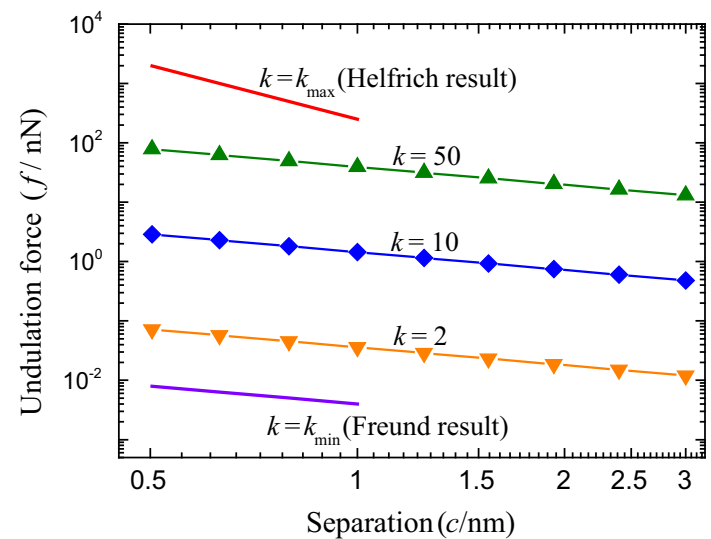

Fig. 3 Computational plots showing lower and upper bounds of undulation force when separation is sufficiently small (here the separation is coarsely three orders of magnitude smaller than the side length of the membrane) for a membrane with $\kappa=5.0 k_{\mathrm{B}} T$ and $L=1.0 \mu \mathrm{m}$. The lower (slop $=-1)$ and upper ( $\operatorname{slop}=-3$ ) lines correspond respectively to Freund's and Helfrich's results

$\lambda_{x}=\lambda_{y}=\lambda_{\min }$, and the energy of the thermal motion in a single direction is roughly $1 /(2 \beta)$. Therefore, the maximum value of the wave number is $m_{\max }=n_{\max }=L / \lambda_{\min }$. According to Eqs. (4) and (5), the value of the wave vector is $q^{2}=2 \pi^{2} / \lambda_{\min }^{2}$, and the maximum amplitude of the wave satisfies $\left|h_{(1,1)}\right|^{2}=c^{2} / \pi^{4}$, and the elastic bending energy of the membrane is calculated as $U=8 \kappa c^{2} / \lambda_{\min }^{2}$. By equating the elastic bending energy to the energy of thermal motion, we obtain the minimum wavelength of the thermal fluctuation of the membrane:

$\lambda_{\min }=4 \sqrt{\kappa \beta} c$.

In terms of the membrane with the given edges of $L \times L$, we note that its maximum wave numbers are $m_{\max }=n_{\max }=$ $L / \lambda_{\min }=2 k_{\max }-1$. Substituting Eq. (15) into Eq. (12) yields the maximum value of the undulation force,

$f_{\max }=\frac{L^{2}}{8 \sqrt{\pi} \kappa \beta^{2} c^{3}} \frac{\omega \exp \left(-\omega^{2}\right)}{\operatorname{erf}(\omega)}$,

where $k_{\max } \gg 1$ is used in Eq. (16).

If the separation is sufficiently small, $\omega \ll 1$ or $c \ll L$, Eq. (16) is written

$f_{\max }=\frac{L^{2}}{16 \kappa \beta^{2} c^{3}}$.

Over the separation range of $c \ll L$, Eq. (17) clearly indicates that the undulation force varies with $c^{-3}$. Equation (17) can readily be written in the form of entropic pressure, $p_{\max }=f_{\max } / L^{2}=1 /\left(16 \kappa \beta^{2} c^{3}\right)$, which is in agreement with the result given by Helfrich and Servuss [21]. Obviously, Eq. (17) forms the upper bound of the undulation force in Eq. (12), as shown in Fig. 3. 


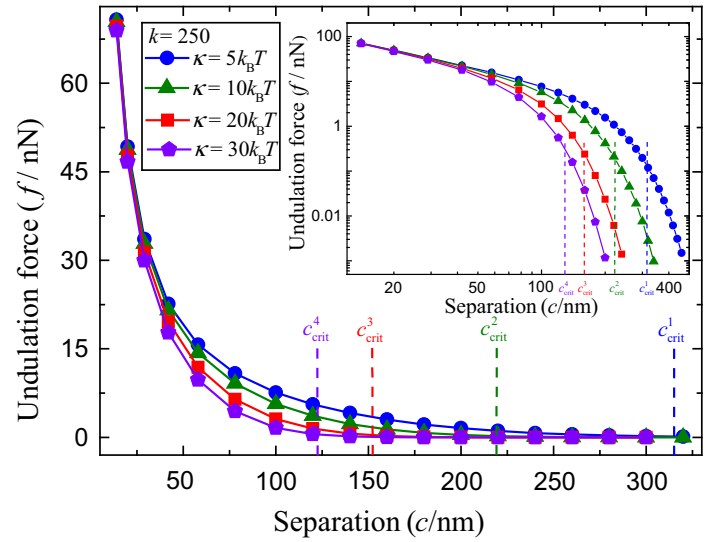

Fig. 4 Computational plots showing critical acting distance of undulation force, $c_{\text {crit }}$. The curves stand for the undulation forces of the membrane with the same wave number and different bending rigidity for $T=300 \mathrm{~K}, L=1.0 \mu \mathrm{m}$, where $c_{\text {crit }}^{1}, c_{\text {crit }}^{2}, c_{\text {crit }}^{3}$, and $c_{\text {crit }}^{4}$ correspond to the different critical distances with the different bending rigidities of membranes. Insert: same data in a double logarithmic coordinate

As stated earlier, the two forms of the undulation force proposed separately by Helfrich [20] and Freund [29] are not contradictory to each other and respectively stand for the upper and lower bounds of the presented result under the condition that the separation is sufficiently small. In fact, Helfrich showed the undulation force affecting the membrane with the large wave number, while Freund gave the undulation force acting on the membrane with a small wave number. Obviously, the result here is intrinsically different from the explanations of the two undulation forces given by Auth and Gompper [32] and Hanlumyuang et al. [33].

Finally, if the separation between membranes is sufficiently large, i.e., $\omega \gg 1$, then the value of the undulation force, from Eq. (12), will approach zero. In fact, according to the nature of the error function, when $\omega \geq 2.0$, the error function will rapidly approach 1 , the error of which is $<0.5 \%$. Because $\Phi\left(\omega_{m n}\right)$ in Eq. (10) is a monotonically decreasing function with respect to $\omega_{m n}$, the undulation force in Eq. (12) will rapidly approach zero. According to Eq. (11), we obtain the distance

$c_{\text {crit }}=\frac{L}{\sqrt{2 \kappa \beta}}$.

Equation (18) shows that there exists a critical separation on the undulation force. Once the separation between membranes is larger than the critical length, the undulation force generated between the membranes will decay and rapidly approach zero (Fig. 4).

\section{Conclusions}

Based on the theories of elasticity and statistical mechanics, an exact integral expression of the undulation force is given in this study. The present result is proved to be in good agreement with existing experiments. Based on the expression of the undulation force, we demonstrate that Helfrich's and Freund's results stand respectively for undulation forces on membranes with large and small wave numbers and correspond respectively to the upper and lower bounds of the presented result when the separation between membranes is sufficiently small. A critical distance between membranes on which the undulation force effectively acts is presented.

Acknowledgments The project was supported by the programs in the National Natural Science Foundation of China (Grants 11232013 and 11472285). L. Li thanks L.B. Freund and R. Lipowsky for insightful discussions. F. Song is very grateful to Y.L. Bai of State Key Laboratory of Nonlinear Mechanics and F.J. Ke of Beijing University of Aeronautics and Astronautics for helpful discussions.

\section{References}

1. Israelachvili, J.N., Wennerström, H.J.: Entropic forces between amphiphilic surfaces in liquids. Chem. Phys. 96, 520-531 (1992)

2. Sharma, P.: Entropic force between membranes reexamined. Proc. Natl. Acad. Sci. USA 110, 1976-1977 (2013)

3. Lin, Y., Freund, L.B.: An lower bound on receptor density for stable cell adhesion due to thermal undulations. J. Mater. Sci. 42, 8904 8910 (2007)

4. Yin, Y.J., Chen, Y.Q., Ni, D., et al.: Shape equations and curvature bifurcations induced by inhomogeneous rigidities in cell membranes. J. Biomech. 38, 1433-1440 (2005)

5. Xu, G.K., Feng, X.Q., Zhao, H.P., et al.: Theoretical study on the competition between cell-cell and cell-matrix adhesions. Phys. Rev. E 80, 011921 (2009)

6. Zhong, Y., Ji, B.H.: How do cells produce and regulate the driving force in the process of migration? Eur. Phys. J. Spec. Top. 223, 1373-1390 (2014)

7. Lindahl, E., Edholm, O.: Mesoscopic undulations and thickness fluctuations in lipid bilayers from molecular dynamics simulations. Biophys. J. 79, 426-433 (2000)

8. Merath, R.J., Seifert, U.: Nonmonotonic fluctuation spectra of membranes pinned or tethered discretely to a substrate. Phys. Rev. E 73, 010401(R) (2006)

9. Ho, I.L.: Fluctuation-induced non-equilibrium transition in a liquid-crystal metastable system. Phys. A 391, 1952-1962 (2012)

10. Loudet, J.C., Dolganov, P.V., Patrício, P., et al.: Undulation instabilities in the meniscus of smectic membranes. Phys. Rev. Lett. 106, 117802 (2011)

11. Safinya, C.R., Roux, D., Smith, G.S., et al.: Steric interactions in a model multimembrane system: a synchrotron X-ray study. Phys. Rev. Lett. 57, 2718-2721 (1986)

12. Beales, P.A., Vanderlick, T.K.: DNA as membrane-bound ligandreceptor pairs: duplex stability is tuned by intermembrane forces. Biophys. J. 96, 1554-1565 (2009)

13. Bouglet, G., Ligoure, C.: Polymer-mediated interactions of fluid membranes in a lyotropic lamellar phase: a small angle X-ray and neutron scattering study. Eur. Phys. J. B 9, 137-147 (1999)

14. Giahi, A., Faris, M.E.A., Bassereau, P., et al.: Active membranes studied by X-ray scattering. Eur. Phys. J. E 23, 431-437 (2007)

15. West, B., Schmid, F.: Fluctuations and elastic properties of lipid membranes in the gel $L_{\beta^{\prime}}$, state: a coarse-grained Monte Carlo study. Soft Matter. 6, 1275-1280 (2010) 
16. Wei, Y.J., Wang, B.L., Wu, J.T., et al.: Bending rigidity and gaussian bending stiffness of single-layered grapheme. Nano Lett. 13, 26-30 (2013)

17. Du, F., Duan, H.L., Xiong, C.Y., et al.: Substrate wettability requirement for the direct transfer of graphene. Appl. Phys. Lett. 107, 143109 (2015)

18. Xu, G.K., Hu, J.L., Lipowsky, R., et al.: Binding constants of membrane-anchored receptors and ligands: A general theory corroborated by Monte Carlo simulations. J. Chem. Phys. 143, 243136 (2015)

19. Xu, G.K., Feng, X.Q., Li, Y.: Self-assembled nanostructures of homopolymer and diblock copolymer blends in a selective solvent. J. Phys. Chem. B 114, 1257-1263 (2010)

20. Helfrich, W.: Steric interaction of fluid membranes in multilayer systems. Z. Naturforsch. Sect. A 33, 305-315 (1978)

21. Helfrich, W., Servuss, R.-M.: Undulation, steric interaction and cohesion of fluid membranes. Il. Nuovo. Cimento. D 3, 137-151 (1984)

22. Kleinert, H.: Fluctuation pressure of membrane between walls. Phys. Lett. A 257, 269-274 (1999)

23. Kastening, B.: Fluctuation pressure of a membrane between walls through five loops. Phys. Rev. E 66, 061102 (2002)

24. Kastening, B.: Fluctuation pressure of a fluid membrane between walls through six loops. Phys. Rev. E 73, 011101 (2006)

25. Bachmann, M., Kleinert, H., Pelster, A.: Strong-coupling calculation of fluctuation pressure of a membrane between walls. Phys. Lett. A 261, 127-133 (1999)

26. Bulut, S., Åslund, I., Topgaard, D., et al.: Lamellar phase separation in a centrifugal field. A method for measuring interbilayer forces. Soft Matter. 6, 4520-4527 (2010)
27. Janke, W., Kleinert, H.: Fluctuation pressure of membrane between walls. Phys. Lett. A 117, 353-357 (1986)

28. Gompper, G., Kroll, D.: Steric interactions in multimembrane systems: a Monte Carlo study. Europhys. Lett. 9, 59-64 (1989)

29. Freund, L.B.: Entropic pressure between biomembranes in a periodic stack due to thermal fluctuations. Proc. Natl. Acad. Sci. USA 110, 2047-2051 (2013)

30. Wennerström, H., Olsson, U., Israelachvili, J.N.: Entropic force between fluid layers. Proc. Natl. Acad. Sci. USA 110, E2944 (2013)

31. Freund, L.B.: Reply to Wennerström et al.: Entropic forces on a confined membrane. Proc. Natl. Acad. Sci. USA 110, E2945 (2013)

32. Auth, T., Gompper, G.: Fluctuation pressure of biomembranes in planar confinement. Phys. Rev. E 88, 010701(R) (2013)

33. Hanlumyuang, Y., Liu, L.P., Sharma, P.: Revisiting the entropic force between fluctuating biological membranes. J. Mech. Phys. Solids. 63, 179-186 (2013)

34. Wennerström, H., Olsson, U.: The undulation force; theoretical results versus experimental demonstrations. Adv. Colloid. Interface. Sci. 208, 10-13 (2014)

35. Freund, L.B.: Fluctuation pressure on a bio-membrane confined within a parabolic potential well. Acta Mech. Sin. 28, 1180-1185 (2012)

36. Gosselin, P., Mohrbach, H., Müller, M.M.: Interface-mediated interactions: entropic forces of curved membranes. Phys. Rev. E 83, 051921 (2011) 